\title{
2-Methoxy-4-Nitroaniline Solubility in Several Aqueous Solvent Mixtures: Determination, Modeling and Preferential Solvation
}

\author{
Huanxin $\mathrm{Li}^{\mathrm{a}}$, Yingnan $\mathrm{Xie}^{\mathrm{a}}$, Zhaoyan $\mathrm{Li}^{\mathrm{a}}$ and Hongkun $\mathrm{Zhao}^{\mathrm{b}, *}$ \\ a School of Biological and Chemical Engineering, Nanyang Institute of Technology, Henan Nanyang 473004, \\ People's Republic of China \\ ${ }^{\mathrm{b}}$ College of Chemistry \& Chemical Engineering, YangZhou University, YangZhou, Jiangsu 225002, People's \\ Republic of China
}

\section{Corresponding author}

Tel: + 86514 87975568; Fax: + 8651487975244 .

*E-mail address: hkzhao@yzu.edu.cn (H.K. Zhao). 


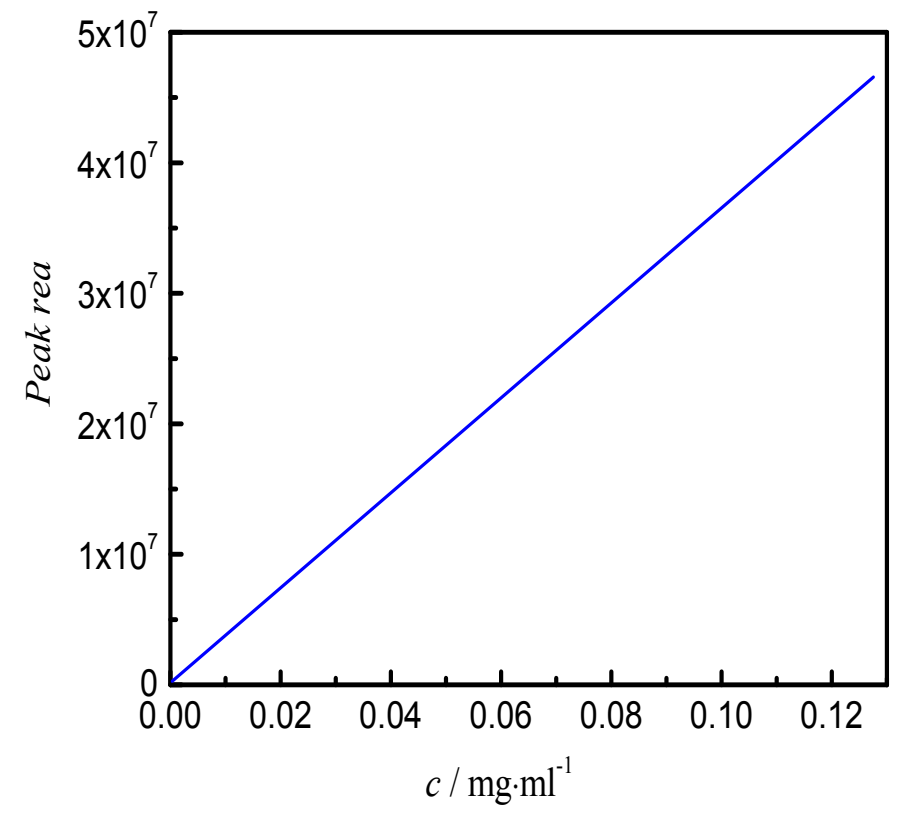

Fig. S1. Relationship between peak area and concentration of 2-methoxy-4-nitroaniline. 


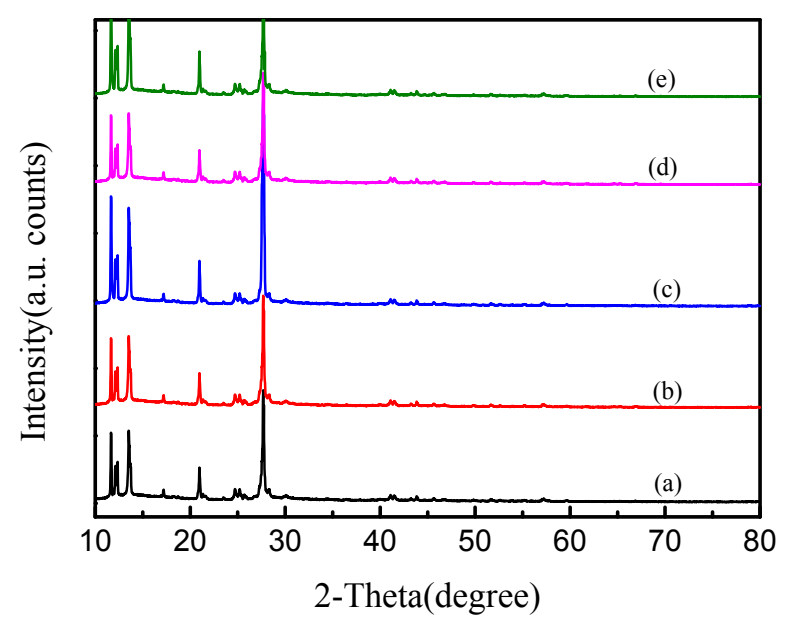

Fig. S2. XRD patterns of 2-methoxy-4-nitroaniline: (a) raw material; (b) equilibrated with $n$-propanol (1) + water (2) mixture; (c) equilibrated with isopropanol (1) + water (2) mixture; (d) equilibrated with EG (1) + water (2) mixture; (e) equilibrated with DMSO (1) + water (2) mixture. 


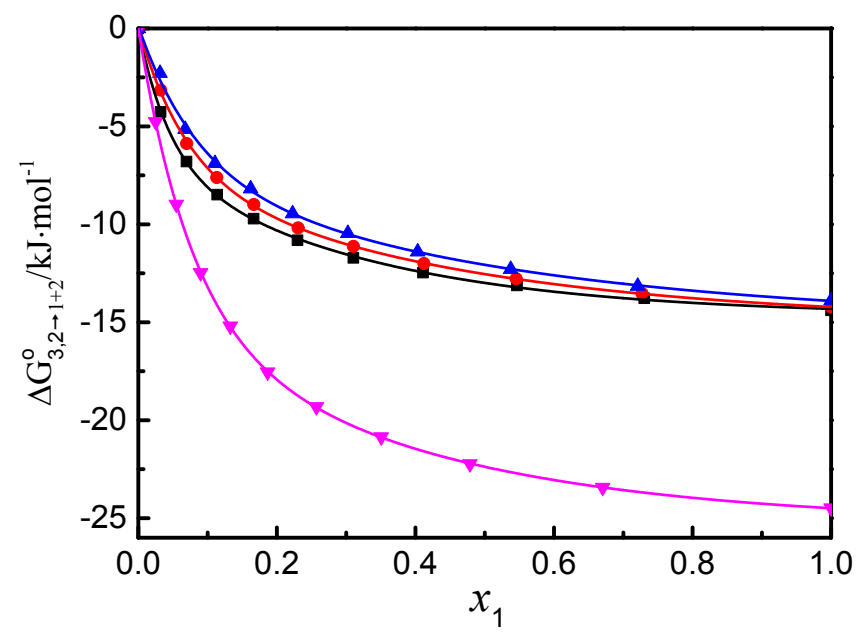

Fig. S3. Gibbs energy of transfer $\left(\mathrm{kJ} \cdot \mathrm{mol}^{-1}\right)$ of 2-methoxy-4-nitroaniline (3) from neat water (2) to $n$-propanol (1) + water (2), isopropanol (1) + water (2), EG (1) + water (2) and DMSO (1) + water (2) mixtures at 298.15 K. m, $n$-propanol (1) + water (2); •, isopropanol (1) + water (2); $\mathbf{\Lambda}$, EG (1) + water (2); $\mathbf{\nabla}$, DMSO (1) + water (2). 
Table S1 Gibbs energy of transfer $\left(\Delta_{\mathrm{tr}} G_{3,2 \rightarrow 1+2}^{\mathrm{o}}, \mathrm{kJ} \cdot \mathrm{mol}^{-1}\right)$ of 2-methoxy-4-nitroaniline from neat water (2) to $n$-propanol (1) + water (2), isopropanol (1) + water (2), EG (1) + water (2) and DMSO (1) + water (2) mixtures at $298.15 \mathrm{~K}$.

\begin{tabular}{|c|c|c|c|c|c|c|c|}
\hline \multicolumn{2}{|c|}{$n$-propanol (1) + water (2) } & \multicolumn{2}{|c|}{ isopropanol (1) + water (2) } & \multicolumn{2}{|c|}{$\mathrm{EG}(1)+$ water $(2)$} & \multicolumn{2}{|c|}{ DMSO (1) + water (2) } \\
\hline$x_{1}{ }^{\mathrm{a}}$ & $\Delta_{\mathrm{tr}} G_{3,2 \rightarrow 1+2}^{\mathrm{o}}$ & $x_{1}^{\mathrm{a}}$ & $\Delta_{\mathrm{tr}} G_{3,2 \rightarrow 1+2}^{\mathrm{o}}$ & $x_{1}{ }^{\mathrm{a}}$ & $\Delta_{\text {tr }} G_{3,2 \rightarrow 1+2}^{\mathrm{o}}$ & $x_{1}^{\mathrm{a}}$ & $\Delta_{\mathrm{tr}} G_{3,2 \rightarrow 1+2}^{\mathrm{o}}$ \\
\hline 0 & 0 & 0 & 0 & 0 & 0 & 0 & 0 \\
\hline 0.03224 & -4.262 & 0.03254 & -3.153 & 0.03128 & -2.280 & 0.02507 & -4.762 \\
\hline 0.06969 & -6.797 & 0.06977 & -5.879 & 0.06763 & -5.144 & 0.05484 & -8.979 \\
\hline 0.1139 & -8.489 & 0.1133 & -7.610 & 0.1108 & -6.887 & 0.08988 & -12.47 \\
\hline 0.1664 & -9.713 & 0.1667 & -8.988 & 0.1622 & -8.180 & 0.1328 & -15.20 \\
\hline 0.2299 & -10.81 & 0.2306 & -10.19 & 0.2228 & -9.453 & 0.1867 & -17.54 \\
\hline 0.3104 & -11.71 & 0.3105 & -11.13 & 0.3024 & -10.46 & 0.2570 & -19.32 \\
\hline 0.4111 & -12.47 & 0.4124 & -12.01 & 0.4032 & -11.40 & 0.3507 & -20.86 \\
\hline 0.5466 & -13.12 & 0.5455 & -12.78 & 0.5376 & -12.30 & 0.4788 & -22.23 \\
\hline 0.7303 & -13.78 & 0.7271 & -13.53 & 0.7208 & -13.16 & 0.6703 & -23.43 \\
\hline 1 & -14.39 & 1 & -14.23 & 1 & -13.89 & 1 & -24.49 \\
\hline
\end{tabular}

${ }^{\text {a }} x$ represents the mole fraction of $n$-propanol (isopropanol, EG or DMSO) in mixed solvents of $n$-propanol (isopropanol, EG or DMSO) $(w)+$ water $(1-w)$ free of the solute. 
Table S2 Coefficients of Eq. (18) (kJ·mol $\left.{ }^{-1}\right)$ applied to Gibbs energy of transfer of 2-methoxy-4-nitroaniline (3) from neat water (2) to $n$-propanol (1) + water (2), isopropanol (1) + water (2), EG (1) + water (2) and DMSO (1) + water (2) solvent mixtures at $298.15 \mathrm{~K}$.

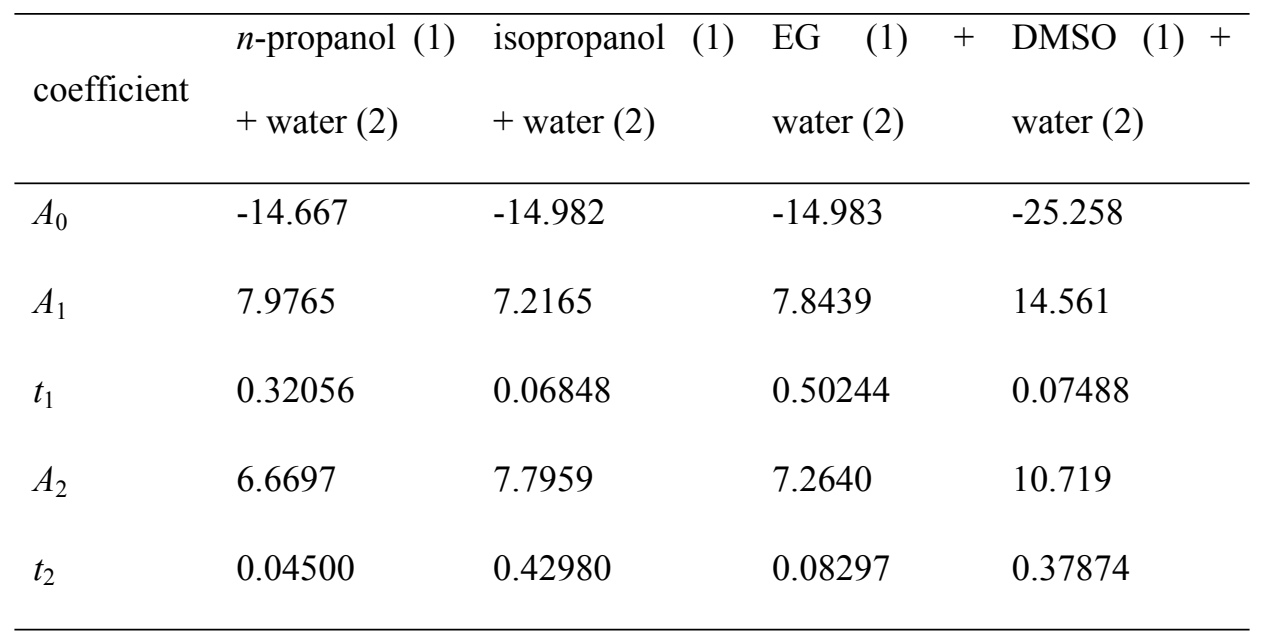


Table S3 Some properties associated to preferential solvation of 2-methoxy-4-nitroaniline (3) in $n$-propanol (1) + water (2) mixtures at $298.15 \mathrm{~K}$.

\begin{tabular}{|c|c|c|c|c|c|}
\hline$x_{1}^{\mathrm{a}}$ & $\begin{array}{l}D \\
\mathrm{~kJ} \cdot \mathrm{mol}^{-1}\end{array}$ & $\begin{array}{l}G_{1,3} \\
\mathrm{~cm}^{3} \cdot \mathrm{mol}^{-1}\end{array}$ & $\begin{array}{l}G_{2,3} \\
\mathrm{~cm}^{3} \cdot \mathrm{mol}^{-1}\end{array}$ & $\begin{array}{l}V_{\text {cor }} \\
\mathrm{cm}^{3} \cdot \mathrm{mol}^{-1}\end{array}$ & $100 \delta x_{1}$ \\
\hline 0.00 & -173.1 & -1383 & -126.4 & 667 & 0 \\
\hline 0.05 & -70.08 & -593.1 & -224.7 & 678 & -4.026 \\
\hline 0.10 & -34.28 & -344.7 & -224.3 & 752 & -2.100 \\
\hline 0.15 & -20.87 & -256.3 & -219.8 & 818 & -0.784 \\
\hline 0.20 & -15.07 & -218.6 & -221.4 & 877 & 0.068 \\
\hline 0.25 & -11.98 & -198.1 & -226.0 & 933 & 0.733 \\
\hline 0.30 & -9.949 & -183.9 & -230.4 & 987 & 1.267 \\
\hline 0.35 & -8.413 & -172.5 & -232.6 & 1039 & 1.651 \\
\hline 0.40 & -7.165 & -162.9 & -231.9 & 1089 & 1.872 \\
\hline 0.45 & -6.120 & -154.8 & -228.5 & 1136 & 1.941 \\
\hline 0.50 & -5.232 & -148.0 & -223.3 & 1182 & 1.890 \\
\hline 0.55 & -4.475 & -142.6 & -217.2 & 1226 & 1.758 \\
\hline 0.60 & -3.829 & -138.4 & -210.8 & 1270 & 1.578 \\
\hline 0.65 & -3.276 & -135.0 & -204.7 & 1313 & 1.375 \\
\hline 0.70 & -2.803 & -132.4 & -199.1 & 1355 & 1.163 \\
\hline 0.75 & -2.398 & -130.4 & -193.7 & 1397 & 0.950 \\
\hline 0.80 & -2.051 & -128.7 & -188.5 & 1439 & 0.736 \\
\hline 0.85 & -1.755 & -127.4 & -182.7 & 1480 & 0.524 \\
\hline 0.90 & -1.502 & -126.4 & -175.7 & 1521 & 0.320 \\
\hline 0.95 & -1.285 & -125.5 & -167.4 & 1561 & 0.139 \\
\hline 1.00 & -1.099 & -125.0 & -158.2 & 1602 & 0 \\
\hline
\end{tabular}


Table S4 Some properties associated to preferential solvation of 2-methoxy-4-nitroaniline (3) in isopropanol (1) + water (2) mixtures at $298.15 \mathrm{~K}$.

\begin{tabular}{|c|c|c|c|c|c|}
\hline$x_{1}{ }^{\mathrm{a}}$ & $\begin{array}{l}D \\
\mathrm{~kJ} \cdot \mathrm{mol}^{-1}\end{array}$ & $\begin{array}{l}G_{1,3} \\
\mathrm{~cm}^{3} \cdot \mathrm{mol}^{-1}\end{array}$ & $\begin{array}{l}G_{2,3} \\
\mathrm{~cm}^{3} \cdot \mathrm{mol}^{-1}\end{array}$ & $\begin{array}{l}V_{\text {cor }} \\
\mathrm{cm}^{3} \cdot \mathrm{mol}^{-1}\end{array}$ & $100 \delta x_{1,3}$ \\
\hline 0.00 & -123.5 & -1026 & -126.4 & 668 & 0 \\
\hline 0.05 & -66.92 & -603.8 & -201.6 & 674 & -4.225 \\
\hline 0.10 & -38.84 & -404.4 & -219.7 & 716 & -3.481 \\
\hline 0.15 & -24.58 & -306.9 & -223.7 & 762 & -2.018 \\
\hline 0.20 & -17.07 & -257.7 & -227.9 & 805 & -0.834 \\
\hline 0.25 & -12.88 & -232.4 & -237.2 & 847 & 0.147 \\
\hline 0.30 & -10.34 & -219.6 & -253.7 & 889 & 1.108 \\
\hline 0.35 & -8.670 & -214.1 & -279.3 & 930 & 2.202 \\
\hline 0.40 & -7.458 & -213.2 & -317.2 & 974 & 3.574 \\
\hline 0.45 & -6.514 & -215.7 & -372.0 & 1020 & 5.380 \\
\hline 0.50 & -5.738 & -220.3 & -448.2 & 1070 & 7.741 \\
\hline 0.55 & -5.079 & -223.6 & -542.3 & 1122 & 10.44 \\
\hline 0.60 & -4.507 & -218.2 & -620.2 & 1168 & 12.22 \\
\hline 0.65 & -4.006 & -197.9 & -616.1 & 1196 & 11.18 \\
\hline 0.70 & -3.562 & -170.3 & -519.0 & 1208 & 7.853 \\
\hline 0.75 & -3.170 & -148.7 & -399.9 & 1220 & 4.669 \\
\hline 0.80 & -2.821 & -136.3 & -307.7 & 1240 & 2.564 \\
\hline 0.85 & -2.511 & -129.8 & -246.6 & 1266 & 1.331 \\
\hline 0.90 & -2.235 & -126.6 & -207.7 & 1295 & 0.629 \\
\hline 0.95 & -1.989 & -125.0 & -182.6 & 1327 & 0.228 \\
\hline 1.00 & -1.771 & -124.2 & -166.0 & 1360 & 0 \\
\hline
\end{tabular}

\footnotetext{
a $x_{1}$ is the mole fraction of isopropanol (1) in the isopropanol (1) + water (2) mixtures free of 2-methoxy-4-nitroaniline (3).
} 
Table S5 Some properties associated to preferential solvation of 2-methoxy-4-nitroaniline (3) in EG (1) + water (2) mixtures at $298.15 \mathrm{~K}$.

\begin{tabular}{|c|c|c|c|c|c|}
\hline$x_{1}{ }^{a}$ & $\begin{array}{l}D \\
\mathrm{~kJ} \cdot \mathrm{mol}^{-1}\end{array}$ & $\begin{array}{l}G_{1,3} \\
\mathrm{~cm}^{3} \cdot \mathrm{mol}^{-1}\end{array}$ & $\begin{array}{l}G_{2,3} \\
\mathrm{~cm}^{3} \cdot \mathrm{mol}^{-1}\end{array}$ & $\begin{array}{l}V_{\text {cor }} \\
\mathrm{cm}^{3} \cdot \mathrm{mol}^{-1}\end{array}$ & $100 \delta x_{1,3}$ \\
\hline 0.00 & -103.2 & -859.8 & -126.4 & 659 & 0 \\
\hline 0.05 & -62.06 & -534.3 & -202.0 & 675 & -3.455 \\
\hline 0.10 & -39.03 & -368.0 & -215.8 & 727 & -2.764 \\
\hline 0.15 & -25.94 & -279.4 & -211.7 & 777 & -1.557 \\
\hline 0.20 & -18.34 & -229.9 & -204.4 & 819 & -0.670 \\
\hline 0.25 & -13.79 & -200.8 & -198.2 & 857 & -0.072 \\
\hline 0.30 & -10.95 & -182.4 & -194.0 & 892 & 0.347 \\
\hline 0.35 & -9.068 & -169.9 & -191.3 & 924 & 0.657 \\
\hline 0.40 & -7.748 & -160.7 & -189.6 & 956 & 0.891 \\
\hline 0.45 & -6.761 & -153.5 & -188.4 & 986 & 1.062 \\
\hline 0.50 & -5.983 & -147.6 & -187.4 & 1017 & 1.173 \\
\hline 0.55 & -5.340 & -142.7 & -186.4 & 1048 & 1.223 \\
\hline 0.60 & -4.793 & -138.5 & -185.3 & 1080 & 1.217 \\
\hline 0.65 & -4.316 & -135.1 & -184.0 & 1112 & 1.159 \\
\hline 0.70 & -3.895 & -132.3 & -182.6 & 1146 & 1.058 \\
\hline 0.75 & -3.519 & -130.1 & -181.2 & 1182 & 0.922 \\
\hline 0.80 & -3.182 & -128.5 & -179.9 & 1220 & 0.761 \\
\hline 0.85 & -2.879 & -127.3 & -178.8 & 1260 & 0.584 \\
\hline 0.90 & -2.605 & -126.7 & -178.2 & 1303 & 0.396 \\
\hline 0.95 & -2.358 & -126.4 & -178.1 & 1348 & 0.201 \\
\hline 1.00 & -2.134 & -126.6 & -179.1 & 1397 & 0 \\
\hline
\end{tabular}

$\mathrm{a} x_{1}$ is the mole fraction of EG (1) in the EG (1) + water (2) mixtures free of 2-methoxy-4-nitroaniline (3). 
Table S6 Some properties associated to preferential solvation of 2-methoxy-4-nitroaniline (3) in DMSO (1) + water (2) mixtures at $298.15 \mathrm{~K}$.

\begin{tabular}{|c|c|c|c|c|c|}
\hline$x_{1}^{\mathrm{a}}$ & $\begin{array}{l}D \\
\mathrm{~kJ} \cdot \mathrm{mol}^{-1}\end{array}$ & $\begin{array}{l}G_{1,3} \\
\mathrm{~cm}^{3} \cdot \mathrm{mol}^{-1}\end{array}$ & $\begin{array}{l}G_{2,3} \\
\mathrm{~cm}^{3} \cdot \mathrm{mol}^{-1}\end{array}$ & $\begin{array}{l}V_{\text {cor }} \\
\mathrm{cm}^{3} \cdot \mathrm{mol}^{-1}\end{array}$ & $100 \delta x_{1,3}$ \\
\hline 0.00 & -222.8 & -1757 & -126.4 & 670 & 0 \\
\hline 0.05 & -124.5 & -1023 & -300.2 & 565 & -15.02 \\
\hline 0.10 & -72.88 & -614.2 & -328.8 & 692 & -7.676 \\
\hline 0.15 & -45.28 & -395.2 & -306.2 & 791 & -2.407 \\
\hline 0.20 & -30.15 & -279.9 & -274.1 & 859 & -0.161 \\
\hline 0.25 & -21.53 & -218.5 & -246.4 & 913 & 0.775 \\
\hline 0.30 & -16.36 & -184.6 & -225.4 & 962 & 1.144 \\
\hline 0.35 & -13.05 & -164.8 & -209.9 & 1008 & 1.259 \\
\hline 0.40 & -10.77 & -152.7 & -198.3 & 1053 & 1.253 \\
\hline 0.45 & -9.104 & -144.9 & -189.3 & 1096 & 1.187 \\
\hline 0.50 & -7.804 & -139.6 & -182.3 & 1139 & 1.091 \\
\hline 0.55 & -6.750 & -135.9 & -176.6 & 1182 & 0.980 \\
\hline 0.60 & -5.870 & -133.3 & -172.1 & 1224 & 0.865 \\
\hline 0.65 & -5.120 & -131.5 & -168.5 & 1266 & 0.752 \\
\hline 0.70 & -4.475 & -130.1 & -165.8 & 1307 & 0.644 \\
\hline 0.75 & -3.915 & -129.1 & -164.0 & 1348 & 0.541 \\
\hline 0.80 & -3.428 & -128.3 & -163.1 & 1389 & 0.444 \\
\hline 0.85 & -3.002 & -127.7 & -163.4 & 1430 & 0.351 \\
\hline 0.90 & -2.630 & -127.2 & -165.4 & 1470 & 0.257 \\
\hline 0.95 & -2.305 & -126.7 & -170.8 & 1509 & 0.151 \\
\hline 1.00 & -2.019 & -126.2 & -184.3 & 1548 & 0 \\
\hline
\end{tabular}

a $x_{1}$ is the mole fraction of DMSO (1) in the DMSO (1) + water (2) mixtures free of 2-methoxy-4-nitroaniline (3). 